\title{
Médiévales
}

Langues, Textes, Histoire

71 | automne 2016

Conflits et concurrence de normes

\section{Marilyn NICOUD, Le Prince et les médecins. Pensée et pratiques médicales à Milan (1402-1476)}

Rome, École française de Rome, 2014, 804 p.

\section{Laetitia Loviconi}

\section{(2) OpenEdition}

Journals

Édition électronique

URL : https://journals.openedition.org/medievales/7932

DOI : $10.4000 /$ medievales. 7932

ISSN : 1777-5892

Éditeur

Presses universitaires de Vincennes

Édition imprimée

Date de publication : 20 novembre 2016

Pagination : 177-179

ISBN : 978-2-84292-565-9

ISSN : 0751-2708

Référence électronique

Laetitia Loviconi, «Marilyn Nicoud, Le Prince et les médecins. Pensée et pratiques médicales à Milan

(1402-1476) », Médiévales [En ligne], 71 | automne 2016, mis en ligne le 29 novembre 2016, consulté le 24 avril 2022. URL : http://journals.openedition.org/medievales/7932 ; DOI : https://doi.org/10.4000/ medievales.7932

Ce document a été généré automatiquement le 24 avril 2022.

Tous droits réservés 


\section{Marilyn NICOUD, Le Prince et les médecins. Pensée et pratiques médicales à Milan (1402-1476)}

Rome, École française de Rome, 2014, 804 p.

\section{Laetitia Loviconi}

\section{RÉFÉRENCE}

Marilyn Nicoud, Le Prince et les médecins. Pensée et pratiques médicales à Milan (1402-1476), Rome, École française de Rome, 2014, 804 p.

1 Fondé sur l'étude maîtrisée et précautionneuse d'une abondance de sources, l'ouvrage de Marilyn Nicoud se focalise sur les médecins ayant évolué au $\mathrm{Xv}^{\mathrm{e}}$ siècle dans le cadre de la cour des Visconti et des Sforza. Il met en lumière quelles furent les relations entre les médecins de cour et le prince, entre université pavesane et cour milanaise, leurs influences réciproques considérées dans leurs dimensions sociales, politiques, culturelles, sans omettre d'examiner les productions écrites des médecins de ce milieu, les modalités et fondements de l'exercice médical, les relations entre médecins, la reconnaissance de l'expertise des médecins et son insertion dans une diversité de contextes politiques et sociaux incluant les questions de santé publique.

2 En un premier chapitre, «La cour et l'université », l'auteure s'intéresse au milieu des médecins ayant œuvré à la cour lombarde au $x^{\mathrm{e}}$ siècle et recrutés en tant que professionnels de la santé, représentants de la médecine savante et souvent issus de l'université pavesane. À ce titre, «l'analyse de ce milieu, de ses composantes et de son rôle ne saurait-elle être menée indépendamment de celle du développement de l'université de Pavie, institution voulue par les seigneurs lombards et constamment soumise à leur tutelle » (p. 23). La forte concentration de praticiens à la cour lombarde est illustrée par l'énumération d'une multitude de médecins extrêmement dense, mais qui rend compte au mieux de la présence ininterrompue d'un personnel médical à la 
cour et qui, par les détails singuliers, démontre les particularités du recrutement dans ce milieu comparativement à d'autres milieux curiaux. Outre la constance du profil universitaire des médecins de cour, ce chapitre révèle l'importance du recrutement local et démontre la continuité ainsi que la longueur des services exercés par un nombre non négligeable de praticiens. Toutefois, la diversité des profils n'est pas tue, qu'il s'agisse de la nature des missions confiées, de la poursuite ou de l'abandon de la carrière professorale durant ses missions. Par-delà cette diversité des cas individuels, l'analyse des sources fait apparaître la permanence d'un groupe de médecins régulièrement présents dans l'entourage proche du prince, de sa parenté, de sa familia, de ses alliés, en charge de leur bien-être. Lieu fréquent de recrutement de ces médecins, le studium lombard est ensuite envisagé plus spécifiquement en des pages qui décrivent le développement de l'université, les modalités de fonctionnement, les structures et enseignements dont elle s'est trouvée pourvue et qui lui ont permis de devenir un centre reconnu de formation professionnelle et un vivier de recrutement de praticiens. Pour l'essentiel bénéfique pour les médecins de cour comme pour le prince, cette situation n'a pas été indemne de toute tension, comme en attestent quelques archives: difficultés pour certains praticiens de concilier carrière universitaire et engagement curial, interventionnisme du pouvoir ducal dans les nominations et dans la détermination des salaires, rivalité interne, mais aussi concurrence entre studia qui révèle «la place occupée par l'université dans la politique ducale, en tant que lieu d'expression d'un rayonnement culturel et intellectuel » (p. 127).

Outre le développement de la médecine au sein du studium et l'émergence d'un milieu professionnel dédié à la santé, le pouvoir ducal favorisa la naissance, la diffusion, la valorisation de savoirs médicaux en dehors de leur strict domaine disciplinaire et de la sphère des praticiens. C'est donc avec la question des "cultures médicales à la cour " que se poursuit l'ouvrage, cultures favorisées par le recrutement local des praticiens au service du prince, par la poursuite de la carrière universitaire choisie par certains d'entre eux, par la circulation des savants et des livres entre cour et université, par l'intérêt marqué par le prince envers les savoirs médicaux et le développement d'une bibliothèque ducale. Si se manifeste une "imprégnation des savoirs médicaux dans d'autres domaines de la culture", "la collaboration de l'ars medica et de ses représentants à l'élaboration d'une culture et d'une fama curiale» (p.156) est étayée par des sources diversifiées. Celles-ci caractérisent l'insertion de connaissances médicales et de préoccupations hygiéniques dans des questions et domaines disciplinaires multiples (politique, architecture, pédagogie notamment). Ainsi, les médecins de cour, loin de n'apporter que leurs soins comme praticiens, constituèrent des moyens, pour le pouvoir ducal, d'établir une image valorisée de la cour aux yeux des puissances voisines, de renforcer des liens politiques avec des alliés auxquels était accordé l'envoi d'un praticien ducal en cas de besoin, de participer à la politique matrimoniale de la famille ducale en se prononçant sur la fertilité des partis envisagés. Malgré l'indiscutable reconnaissance ainsi obtenue par les médecins, Marilyn Nicoud souligne combien fut également cruciale pour eux l'écriture d'une ou plusieurs œuvres afin d'établir fermement leur identité professionnelle et de bénéficier d'une fama intellectuelle. Le tableau des auteurs et œuvres qu'on peut rattacher au studium lombard donne à voir les aspects singuliers et certaines évolutions des écrits médicaux communes à la fin du Moyen Âge, leurs particularités dans le milieu lombard, les voies de leur réception et de leur diffusion sous formes manuscrite et imprimée, les témoignages offerts par ces ouvrages: besoins et attentes des milieux de cour, 
fondements et conditions de l'exercice médical, particulièrement auprès des élites sociales.

4 C'est autour du corps de ces élites, et particulièrement celui de souverains, que se noue une relation entre patient et praticien, sur ce corps que se réalise une observation génératrice de savoirs et d'écrits, sur ce corps que les médecins de cour mettent en œuvre leur pratique, comme l'illustre et le prouve le troisième chapitre «Corps en paroles » qui s'appuie sur l'étude croisée d'écrits rédigés par une grande variété de correspondants, intéressés par la santé du prince et de ses proches pour des raisons diverses. Le chapitre se décline en axes d'étude complémentaires : examiner ce que donnent à voir les écrits sur les relations entre praticiens et patients (ici en milieu de cour), envisager le fond proprement médical et la forme, les procédés rédactionnels mobilisés. Le médecin de cour apparaît comme observateur des corps et prescripteur dans les domaines préventif et thérapeutique; et, malgré de possibles réticences de leurs patients, voire de nécessaires négociations, ils ne voient jamais niée leur expertise dans le domaine de la santé et acquièrent le statut d' "expert des eaux thermales " (p. 290). Dans ce milieu de cour émerge ainsi l'image d'une relation patient-praticien fondée sur la confiance et l'obéissance du patient, «les critiques à l'encontre des médecins sont peu nombreuses dans l'ensemble, surtout lorsque l'information émane de la cour. Elles ne regardent jamais leurs capacités et leurs savoirs, mais plutôt leurs éventuelles absences ou négligence »(p.307). Parfois requis auprès des enfants du couple ducal, pour des préoccupations sanitaires, le médecin de cour peut alors également être consulté sur des aspects moraux et pédagogiques. Au gré de ces diverses activités, et particulièrement lors de la délivrance de soins, les médecins de cour sont amenés à rédiger de fréquents courriers dont les formes, intentions et contenus sont envisagés en lien avec l'exercice médical en milieu curial. L'analyse des correspondances révèle un exercice médical de cour fréquemment basé sur une collaboration entre confrères dont sont étudiés les modalités et le fruit, la collatio. Sans nier que des divergences (très rarement rapportées) aient certainement émaillé la phase de délibération, $\mathrm{M}$. Nicoud montre combien l'expression de la collatio transmet l'image d'une unité de jugement et de cohésion entre médecins de cour. La correspondance émanant des médecins et destinée au couple ducal restitue le suivi médical à partir de faits sélectionnés, ordonnés, mis en mots de façon réfléchie pour informer, susciter la confiance, justifier les prescriptions. L'auteure conclut qu'« en justifiant ainsi une intervention médicale sur tous les instants de la vie, en inscrivant pleinement la conservatio sanitatis au sein de l'acte médical, d'un côté ils cherchent à réduire la part d'aléatoire, si ce n'est le danger, propre à l'action thérapeutique, de l'autre à s'imposer comme les maîtres des corps » (p. 380-381). Si l'on peut s'interroger sur le caractère avéré de cette recherche de statut de " maîtres des corps », les données exposées sont très convaincantes à l'égard de l'étendue et de la reconnaissance croissantes des domaines d'expertise des médecins de cour.

5 Parmi les domaines d'expertise strictement médicale figure le champ de la santé publique, en particulier en un siècle où sévit la peste, comme le montre le dernier chapitre, «Les médecins et la peste ». Membres primordiaux du système d'information et de déclaration des nouveaux cas suspects, les médecins, sur la base des étiologies supposées, tentaient par ailleurs de reconstruire le parcours de l'épidémie, voire d'en prévoir le cheminement futur, pour favoriser la prise de mesures préventives, cruciales en raison d'un échec des approches thérapeutiques. L'engagement médical contre l'épidémie se traduit sur deux versants concrétisés dans des ouvrages médicaux (traités 
sur la peste) et des correspondances, témoins de l'exercice médical. Présents sur le terrain ou informés par des courriers dont ils sont les destinataires, les praticiens délivrent leurs diagnostics sur les cas suspects à l'issue de délibérations parfois collectives et basées sur le récit des histoires particulières et des visites réitérées pour tenter de lever tout doute, sur une réflexion prudente et l'inventaire des arguments pro et contra. Assurément, cette prudence se justifie doublement. Malgré quelques traces de divergences entre avis médical et décisions des autorités, c'est en effet sur la base de l'expertise médicale que les autorités se prononcent sur d'éventuelles mesures d'isolement ou d'éloignement, synonymes de mort sociale et moyens d'endiguer l'épidémie. Par ailleurs, la prudence est motivée par des considérations sémiologiques. Seul le bubon apparaît comme signe suffisant de peste et, en son absence, le diagnostic doit s'appuyer sur plusieurs signes dont la présence est fluctuante et qui sont parfois équivoques.

6 M. Nicoud fournit donc une étude rigoureuse, riche et éclairante sur la figure du médecin de cour, et révélatrice de multiples facettes quant aux relations existant entre les médecins de cour, le prince en tant que patient et détenteur du pouvoir, le studium pavesan, le milieu intellectuel curial, abordant les dimensions culturelles, politiques, sociales, n'omettant ni l'examen des théories médicales, ni celles des pratiques médicales et de leur interaction avec les fondements conceptuels. En outre, l'analyse s'accompagne d'une prudence raisonnée, eu égard aux lacunes archivistiques, et, chaque fois que cela est possible, une comparaison avec la période antérieure et les autres milieux de cour vient compléter le propos. Cette analyse est accompagnée d'une série de dossiers établis « à partir de dépouillement de catalogues et de publications, d'éditions de sources et d'informations sur les manuscrits des ouvrages lombards " (p. 481), ainsi que d'une large bibliographie compilée de façon très structurée. 